\title{
Genetics of Brain and Cognitive Aging: Introduction to the Special Issue of Neuropsychology Review
}

\author{
Nancy L. Pedersen • Lotte Gerritsen
}

Received: 10 February 2015 / Accepted: 10 February 2015 / Published online: 21 February 2015

(C) Springer Science+Business Media New York 2015

Ever since Sir Francis Galton expounded on the role of heredity in human variation, from mental abilities to physical characteristics, psychologists, biologists, and geneticists have pursued scholarly exploration into the role of nature and nurture for human traits. Some traits that are more somatically defined, such as morphological characteristics (height) were assumed to be more heritable than other traits, such as human behavior, for which the role of genetic influences has at times been fiercely debated. During the last 35 years, there has been a growing appreciation that genetic variation is relevant for understanding individual differences in human traits, from morphological characteristics to behavioral traits. Indeed, the focus has moved from quantifying the relative contribution of genetic influences, i.e. estimating heritability, to identifying which genes are involved in pathways that impinge on human traits in order to understand the genetic architecture.

This issue of Neuropsychology Review is a timely sequel to the previous special issues on Aging and Dementia (Volume 24, Issue 3) and on Development of Human Brain Structure and Function (Volume 20, Issue 4). The papers in the current issue review the role of genes on the full range of brain development, morphology and function from childhood through late life, human communication, cognitive abilities, and dementia.

The current issue of the journal touches on a variety of methodologies available to understand the genetics of brain and cognitive aging. The utility of comparative genomics for understanding the "ancient genetic foundations" of human communicative abilities is elucidated by Graham, Derizoitis, and Fisher. Moving beyond an evolutionary perspective, they

N. L. Pedersen $(\bowtie) \cdot$ L. Gerritsen

Department of Medical Epidemiology and Biostatistics, Karolinska Institutet, Box 281, Stockholm SE-171 77, Sweden

e-mail: nancy.pedersen@ki.se also describe a number of other strategies that have been fruitful for understanding genetic factors in communicative abilities, such as taking a developmental perspective and studying developmental communication disorders and linkage studies to identify specific candidate genes followed by karyotyping to identify chromosomal aberrations in rare disorders. As in several of the other reviews in this issue, they also describe the utility of twin studies for evaluating heritability, endophenotypes through neuroimaging, experimental paradigms and animal models to understand the function of specific genes, and the role of genome wide association studies (GWAS).

The following three reviews - by Jansen et al., Papenberg et al., and Strike et al.- all have in common a focus on genetics of brain structure and function. Jansen et al. summarize what twin studies tell about the heritability of brain development, morphology and function, contrasting findings from children, adults and the elderly. Heritabilities of brain structure are generally substantial, and cross-sectional comparisons suggest slight increases from childhood to adulthood. Longitudinal studies are needed to understand better the heritability of changes in measurements of structure or function.

In the next review, Papenberg et al. provide an in-depth appraisal of select candidate genes and functional imaging, with a special emphasis on old age. They put findings of age related magnification of genetic effects into the perspective of a theoretical framework, the resource-modulation hypothesis, suggesting that genetic influences become stronger when brain resources decrease with age.

Strike et al. expand on twin/family findings such as those summarized by Jansen et al., providing evidence for the overlap in genetic influences among brain structures and between structures and cognition. Furthermore, they provide a comprehensive discussion of the potentials and pitfalls of candidate gene and genome-wide studies of brain morphology, 
including summaries of the most recent findings and their consequences for understanding brain genetics.

Several of the reviews have addressed heritability; however, Reynolds \& Finkel empirically evaluate a number of issues regarding the heritability of cognitive aging. In contrast to most behavioral genetic summaries of age-related patterns of heritability for general cognitive abilities, they provide metaanalyses for specific cognitive abilities, and demonstrate that the domains of cognitive function that show patterns of increasing heritability up to age 70 are those that have been most closely associated with structural changes in specific brain regions. Further, comparisons of monozygotic:dizygotic (MZ:DZ) twin correlation ratios provide insights into the source of "missing heritability," both gene-gene and gene by environment (GXE) interactions contribute to the discrepancies between twin-based and GWAS based findings.

Finally, Ferencz and Gerritsen address the genetics and underlying pathology of dementia. In contrast to many reviews, they look not only at Alzheimer's disease (AD), but also the other common dementia types: vascular dementia (VaD), frontotemporal demential (FTLD), and Lewy-body dementia (LBD), and the overlap in major underlying pathologies. This review exemplifies the need to go further than finding simple associations. Indeed, as GWAS data grow, possibilities to do pathway analyses that can inform overlap in pathologies will be warranted. However, this is likely to take a long time as sufficient numbers of well-characterized, non$\mathrm{AD}$ cases will take some time to accrue.

What is one to make of the wealth of information provided by these reviews?

1) Age matters! Not only do the phenotypes of interest change with age, but also the genetic contributions, whether assessed as heritability estimates or associations with candidate or genome-wide genetic variants, may differ across the life span. Recognition of this will help advancements in understanding mechanisms, particularly when considered in a longitudinal framework.

2) The genetic toolbox is rich; some tools may seem rusty, others are undergoing refinement, and hybrids may prove to serve more needs. Twin and family based studies have provided a wealth of information on the relative importance of genetic influences, and thus some indication of the statistical power for discovering the causal genes for a trait. Further extensions of twin studies to multivariate issues (e.g., genetic correlations between structure and cognition), moderation models, and tests of GXE are valuable, under-utilized components the genetic armamentarium, and uniquely suitable for including environmental information. Both candidate gene and GWAS approaches are affected, albeit in different ways, by power issues, and potentially by publication biases. There are technical issues in high-throughput genotyping platforms that may contribute to much of the "missing" or "hidden" heritability, such as inability to include or "tag" relevant genetic variants (insertions, deletions, inversions and translocations) or lack of coverage of rare variants. Furthermore, what may be of greatest relevance to brain-based phenotypes (particularly function) and behaviors, such as cognition, are regulatory elements and promotor regions, the function of which is best understood through expression studies. Reliance on blood/ leucocyte-based genotyping precludes tissue relevant expression and epigenetic analyses.

3) The causal nexus is complex. Complex phenotypes, such as behaviors, communication, brain function, and brain structure, result from a intricate nexus of multiple inputs from genetic and environmental sources. There are multiple processes, from DNA to RNA to protein products to cells to synaptic clefts, all of which may be impinged upon by other components in the system (McClearn 2006). Thus, epigenetic influences, gene-gene, and gene-environment interactions and correlations are all complexities that represent challenges to understanding the genetics of brain and cognitive aging.

The complexity is compounded by the nature of the phenotypes of interest and methods for assessing them. The brain has been considered an endophenotype that is an intermediary between genes and behavior. Thus, by understanding the genetics of the brain we might be able to better understand the etiology of neuropsychiatric diseases and neurobehavioral development (Mayer-Lindenberg and Weinberg 2006). However, due to the large number of features of the brain and similar density of genomic information, there are considerable challenges to overcome problems of multiple comparisons, harmonization, and differences across platforms, all of which are even greater for functional neuroimaging. New methods and strategies are therefore necessary to accommodate the big data that will be generated by combining the genome with the brain. Nevertheless, we are entering an exciting era that surely will advance our understanding of the complex causal nexus for the brain and cognitive aging.

Acknowledgments During the writing of this work, Dr. Nancy Pedersen received support from NIH grant AG037985, the Swedish Research Council 521-2013-8689, and the Swedish Research Council for Health, Working Life and Welfare 2013-2292.

\section{References}

Mayer-Lindenberg A., \& Weinberg D. R. (2006). Intermediate phenotypes and genetic mechanisms of psychiatric disorders. Nature Reviews Neuroscience, 6, 818-827.

McClearn G. E. (2006). Contextual genetics. Trends in Genetics, 22(6), 314-319. 